\title{
A model for the phase stability of arbitrary nanoparticles as a function of size and shape
}

\author{
A. S. Barnard ${ }^{\text {a) }}$ \\ Center for Nanoscale Materials, Argonne National Laboratory, Argonne, Illinois 60439 \\ P. Zapol ${ }^{\text {b) }}$ \\ Center for Nanoscale Materials, Argonne National Laboratory, Argonne, Illinois 60439 \\ and Materials Science and Chemistry Divisions, Argonne National Laboratory, Argonne, Illinois 60439
}

(Received 16 February 2004; accepted 2 June 2004)

\begin{abstract}
A thermodynamic model describing relative stability of different shapes for nanoparticles as a function of their size was developed for arbitrary crystalline solids and applied to group IV semiconductors. The model makes use of various surface, edge and corner energies, and takes into account surface tension. Approximations and importance of each term of the model were analyzed. The predictions for clean and hydrogenated diamond nanoparticles are compared to explicitly calculated density functional results. It is shown that diamond nanocrystal morphology is markedly different from silicon and germanium. (C) 2004 American Institute of Physics.
\end{abstract}

[DOI: $10.1063 / 1.1775770]$

\section{INTRODUCTION}

Nanoparticle shape can be a factor responsible for their chemical, optical and electronic properties. For instance, nanodiamond morphology has been found to affect its phase stability (e.g., nanocarbon ${ }^{1,2}$ ), and silicon nanoparticle shape is found to affect its luminescence spectrum. ${ }^{3,4}$ Recently quantum confinement in semiconductor nanoparticles has been shown to be shape dependent. ${ }^{5}$ The systematic description of shape evolution with the particle size is extremely difficult, both experimentally and theoretically. Therefore, more often than not, nanoparticles are described as being spherical. Accurate explicit computational studies of nanoparticles, such as recent studies of diamond nanocrystals and nanowires, ${ }^{1,2} \mathrm{Si}$ (Ref. 6) and Ge (Ref. 7) nanoparticles, are very demanding and difficult to extend to sizes above 1-3 $\mathrm{nm}$. It is desirable to compare stability of all nanoparticle morphologies from a limited set of experimental or theoretical data containing bulk, surface, and edge properties. This paper describes a thermodynamic model based on the Gibbs free energy of an arbitrary nanoparticle that may be in principle applied to any system to make a prediction of the energetically preferred shape as a function of the particle size.

The goal of this study is to determine if the relative stability of different morphologies can be quantitatively described using this thermodynamic treatment, by comparing the model predictions with available density functional results for $\mathrm{Si}, \mathrm{Ge}$, and diamond nanoparticles. The surface energies for these three examples are taken from previous density functional calculations in local density approximation (LDA) with ultrasoft pseudopotentials of Stekolnikov et al. ${ }^{8}$ We begin by estimating the size regimes in which corner and edge effects will be expected to be significant. Then we

a)Electronic mail: amanda.barnard@anl.gov

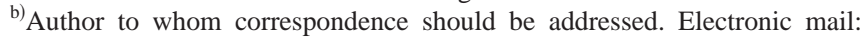
zapol@anl.gov evaluate the influence of the accuracy of surface energies on the results. We also compare two different approaches to evaluation of bulk strain energy, to see which method is valid in the present context. The first approach by Stoneham ${ }^{9}$ explicitly incorporates particle shape, whereas the second (and widely used) Laplace-Young approach assumes a spherical shape.

The evolution of previous work relating to crystal morphology began with approaches independent on crystal size, which are valid at the macroscale. Relative stability of different crystal shapes was first addressed in the classical work of Wulff, ${ }^{10}$ based on the minimization of the total surface energy. Detailed criteria of thermodynamic stability of faceted and "rounded" shapes were further developed by Herring. ${ }^{11}$ There is extensive literature dedicated to the description of macroscopic crystal shapes under different conditions (for example, Refs. 12 and 13). However, to properly describe nanocrystal shape for metals and semiconductors in thermodynamic equilibrium as a function of crystal size, volume-dependent elastic terms should be included in the free energy expressions. The properties were addressed by Cleveland and Landman, ${ }^{14}$ who considered the total energy of different shapes as a sum of bulk and surface energies and a uniform bulk strain, and predicted stable shapes of nickel in agreement with atomistic simulations. Further, a review by José-Yacamán et al. ${ }^{15}$ of shape and stability for metal nanoparticles in the size range of $1-100 \mathrm{~nm}$ discusses both theoretical and experimental results as well as mechanisms of stress release in larger nanoparticles. They concluded that truncation leads to the preferred shapes of small nanoparticles (up to $5 \mathrm{~nm}$ ) but for larger sizes internal stresses and consequent defect formation dominate nanoparticle structures. Moll, Scheffler, and Pehlke ${ }^{16}$ studied the influence of GaAs surface tension on the equilibrium shape of InAs quantum dots, using density functional calculations of edge energies, surface energies and surface tensions combined with 


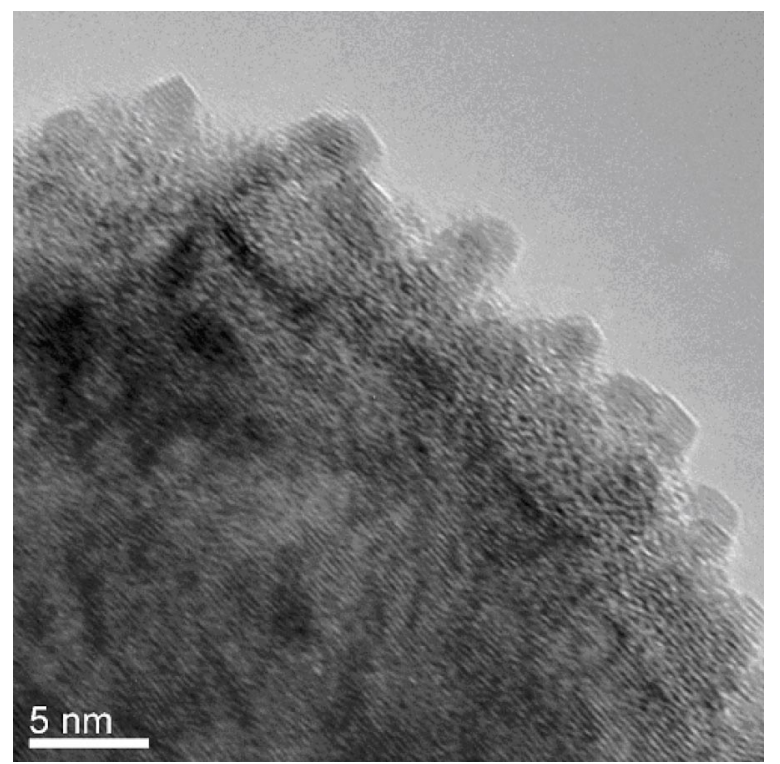

FIG. 1. Pointed metal needle (formed by electrochemically etching $125 \mu \mathrm{m}$ diameter molybdenum wire) with electrophoretically deposited nanodiamond particles; faceted shape of particles is apparent. Courtesy T. Tyler (Ref. 19) and J. Hren, Materials Science and Engineering, North Carolina State University.

finite element calculations of elastic energy. As a result of different scaling behavior of surface energies and elastic energy, the InAs island shape was found to be dependent on the volume of the particles.

Experimental difficulties in determining the most stable nanocrystal shape include size and isomer distributions, chemical environment and presence of thermodynamically nonequilibrium shapes. For example, a tetrakaidecahedron (or truncated octahedron) shape with rounded corners was obtained for $\mathrm{Si}$ voids smaller than $50 \mathrm{~nm}$ by Eaglesham et al. ${ }^{17}$ However, despite the fact that different shapes have been observed for semiconductor nanocrystals, ${ }^{18}$ such as the faceted nanodiamond crystals shown in Fig. 1 (obtained using a JEOL 2010F transmission electron microscope), ${ }^{19}$ a systematic interpretation of experimental results remains elusive.

\section{THEORETICAL FORMALISM}

\section{A. Gibbs free energy for arbitrary nanoparticles}

A complete treatment of the free energy of a nanoparticle should include contributions not just from the bulk and surface of the particle, but also from the edges and the corners. In general, when determining the comparative stability of various shapes the latter contributions will be significant only for small clusters.

As mentioned above, the model proposed here is based on the Gibbs free energy. For a given nanoparticle of material in a phase $x$, the free energy may be expressed as a sum of contributions from the particle bulk, surfaces, edges, and corners, such that,

$$
G_{x}^{o}=G_{x}^{\text {bulk }}+G_{x}^{\text {surface }}+G_{x}^{e d g e}+G_{x}^{\text {corner }} .
$$

The first term, $G_{x}^{\text {bulk }}$ is defined as the standard free energy of formation,

$$
G_{x}^{b u l k}=\Delta_{f} G_{x}^{o}(T),
$$

which is dependent on the temperature $T$. The second term $G_{x}^{\text {surface }}$ may be expressed in terms of the surface free energy $\gamma_{x}$ and the total molar surface area $A$ (which in turn may be written in terms of the molar mass $M$ and density $\rho_{x}$ of the material in a phase $x$ ), and the surface to volume ratio $q$ :

$$
G_{x}^{\text {surface }}=A \gamma_{x}(T)=\frac{M}{\rho_{x}} q \sum_{i} f_{i} \gamma_{x i}(T),
$$

where $\gamma_{x i}(T)$ is the surface free energy of facet $i$ and $f_{i}$ is a weighting factor defined so that,

$$
\sum_{i} f_{i}=1
$$

The surface free energy explicitly depends on the crystallographic orientation of the surface, and on the properties of $x$. Using the same approach, the energy associated with an edge $G_{x}^{\text {edge }}$ may be expressed in terms of the edge free energy $\lambda_{x}$ and the total length $L$ of the edges $j$,

$$
G_{x}^{e d g e}=L \lambda_{x}(T)=L \sum_{j} g_{j} \lambda_{x j}(T),
$$

with $g_{j}$ as the weighting factor,

$$
\sum_{j} g_{j}=\sum_{j} \frac{l_{j}}{L}=1 .
$$

The length of each edge $l_{j}$ may be determined geometrically, and the edge to volume ratio $p$ defined so that, once again,

$$
G_{x}^{e d g e}=\frac{M}{\rho_{x}} p \sum_{j} g_{j} \lambda_{x j}(T) .
$$

Similarly, the energy associated with a corner, $G_{x}^{\text {corner }}$, may be expressed in terms of the corner free energy $\varepsilon_{x}$ and the total number $W$ of corners $k$, such that

$$
\begin{aligned}
G_{x}^{\text {corner }} & =W \varepsilon_{x}(T)=W \sum_{k} h_{k} \varepsilon_{x k}(T), \\
& \Rightarrow G_{x}^{\text {corner }}=\frac{M}{\rho_{x}} w \sum_{k} h_{k} \varepsilon_{x k}(T),
\end{aligned}
$$

with $w$ the corner to volume ratio and $h_{k}$ the weighting factor. The energies of the edges $\lambda_{x j}$ and the corners $\varepsilon_{x k}$ will, of course, depend on the crystallographic orientation of the intersecting surfaces, and on the properties of phase $x$.

Therefore, Eq. (1) becomes

$$
\begin{aligned}
G_{x}^{o}= & \Delta_{f} G_{x}^{o}(T)+\frac{M}{\rho_{x}}\left[q \sum_{i} f_{i} \gamma_{x i}(T)+p \sum_{j} g_{j} \lambda_{x j}(T)\right. \\
& \left.+w \sum_{k} h_{k} \varepsilon_{x k}(T)\right] .
\end{aligned}
$$




\section{B. Effective pressure and surface tension}

However, this does not account for the effects of surface tension on the particle, which are significant at the nanoscale and may not be ignored. The surface tension produces an effective pressure $P_{\text {eff }}$ on the particle, which may be addressed by introducing the resulting (usually compressive) volume dilation $e$,

$$
\frac{\Delta V}{V}=e=P_{\mathrm{eff}} \beta,
$$

which is dependent on the surface tension $\sigma$ and the material compressibility $\beta$. The effect of this dilation will be to reduce the molar volume. Therefore the model, including the effective pressure becomes

$$
\begin{aligned}
G_{x}^{o}= & \Delta_{f} G_{x}^{o}(T)+\frac{M}{\rho_{x}}(1-e)\left[q \sum_{i} f_{i} \gamma_{x i}(T)\right. \\
& \left.+p \sum_{j} g_{j} \lambda_{x j}(T)+w \sum_{k} h_{k} \varepsilon_{x k}(T)\right] .
\end{aligned}
$$

In general, the volume dilation due to $\sigma$ may be approximated using such expressions as the Laplace-Young equation,

$$
P_{\text {eff }}=\frac{2 \sigma}{R},
$$

where $R$ is the mean radius of the particle, so that

$$
e=\frac{2 \beta \sigma}{R} \text {. }
$$

This approach assumes that the particles are spherical, and that the surface tension is independent of orientation. The pressure induced at any point of the surface is directed to the center of curvature and is proportional to the curvature at that point. Although widely used, the applicability of this approach to realistic crystalline nanoparticles may not be ideal, especially within a model designed to examine equilibrium shapes, which might be far from spherical.

A more general expression derived by Stoneham ${ }^{9}$ for facetted crystals, suggests that the surface tension be represented by a force $\sigma$ nd $l$ exerted (normal to a facet edge) on an element $\mathrm{d} l$ of a facet edge. If the force is constant along a given edge, then this may be replaced by the contribution of the total force acting at the center of the edge, in the direction of the direction cosines. For isometric systems, Stoneham showed that the volume dilation reduces to

$$
e=\frac{\beta}{V} \mathcal{G},
$$

where $\mathcal{G}$ is the virial, defined in the direction $\alpha$ by the center $R_{c J \alpha}^{I}$ of edge $J$ of facet $I$, with length $\Lambda_{J}^{I}$ and direction cosine $\Theta_{\alpha}^{I J}$,

$$
\mathcal{G}=\mathcal{G}_{\alpha}^{I J}=R_{c J \alpha}^{I} \Lambda_{J}^{I} \Theta_{\alpha}^{I J} \sigma_{I} .
$$

For example, in the case of a cube of side length $2 a, e$ $=2 \beta \sigma / a$, for an octahedron of length $2 b$ between opposite vertices $e=3 \sqrt{ } 3 \beta \sigma / 2 b$, and for a sphere this was shown to reduce to the Laplace-Young equation [Eq. (14)].
Although it is known that $\sigma=\gamma+A(\partial \gamma / \partial A)$ often, for the case where the change of $\gamma$ with $A$ is small, the approximation $\sigma=\gamma$ is made. Rigorous treatments of surface tension should include anisotropy, resulting in replacement of surface tension by a stress tensor. This can be calculated in a straightforward manner using first-principles methods. Anisotropy of volume expansion may also be included in the expressions. In the following application of our proposed method, we explore the common approximation described above $(\sigma=\gamma)$ for $\mathrm{Si}$.

It should also be noted that the set of surface energies used here ${ }^{8}$ has been calculated at $T=0$, so that the Gibbs free energy obtained from Eq. (12) is (in this case) equivalent to the enthalpy of formation.

\section{RESULTS AND DISCUSSION}

\section{A. Edge and corner energies}

The importance of $\lambda_{x j}$ and $\varepsilon_{x k}$ in the total free energy of the system is largely unknown. To examine the significance these terms in Eq. (12), we first consider the fraction of atoms within a nanoparticle that reside on edges. For a cubic diamond, Si or Ge nanocrystal of approximately $\sim 200$ atoms, the fraction of the total atoms on the edges is $\sim 9 \%$. For a cubic crystal of $\sim 10^{3}$ atoms, the fraction of atoms on the edges decreases to $\sim 4 \%$, to $\sim 1 \%$ when for a crystal of $\sim 10^{4}$ atoms, and still further to $\sim 0.3 \%$ when for a crystal of $\sim 10^{5}$ atoms. For the latter sizes this is significantly less than the fraction of atoms residing on the facet surface $(\sim 5 \%$ for a $\sim 10^{4}$ atom and $\sim 3 \%$ for a $\sim 10^{5}$ atom nanocrystal), indicating that the contribution from the surfaces will dominate at sizes when the total edge energy is small compared to the total surface energy.

Literature data on edge energies are very scarce (note that we differentiate edge energies as defined in Eq. (7) from the step energies, also called step-edge energies, which have been addressed quite extensively). Experimental estimates for $\mathrm{Si}$ edge energies in [110] direction vary from $1.0 \times 10^{-11} \mathrm{~J} / \mathrm{m}$ for $(100)$ facets to $5.7 \times 10^{-11} \mathrm{~J} / \mathrm{m}$ for (111) facets. ${ }^{17}$

Experimental information on diamond and germanium edge energies is not available, however we can make approximate estimates of their importance using available step energies. The estimate is purely qualitative since a single step energy represents edge energy if only nearest neighbor interactions are taken into account. In the case of diamond, for steps parallel to [110] on (111) surface, step energies were calculated ${ }^{20}$ using LDA to be $1.6 \times 10^{-9} \mathrm{~J} / \mathrm{m}$ for clean surface and $-1.7 \times 10^{-9} \mathrm{~J} / \mathrm{m}$ for hydrogenated surface ("ledge energies" in Ref. 20). For single steps parallel to [110] on (100) diamond surface, the step energies were calculated $^{21}$ using non-self-consistent Harris functional to be $8 \times 10^{-11} \mathrm{~J} / \mathrm{m}$ for $\mathrm{S}_{A}$ step and $7 \times 10^{-10} \mathrm{~J} / \mathrm{m}$ for $\mathrm{S}_{B}$ step. It must be noted here that these step energies vary significantly with step height ${ }^{21}$ and the energy of the exposed minifacet normal to the surface is not taken into account. For germanium (001), the step energies for $S_{A}$ and $S_{B}$ steps were experimentally determined by Zandvliet ${ }^{22}$ (see also Zandvliet ${ }^{23}$ ). The zero-temperature values from Fig. 3 of this 

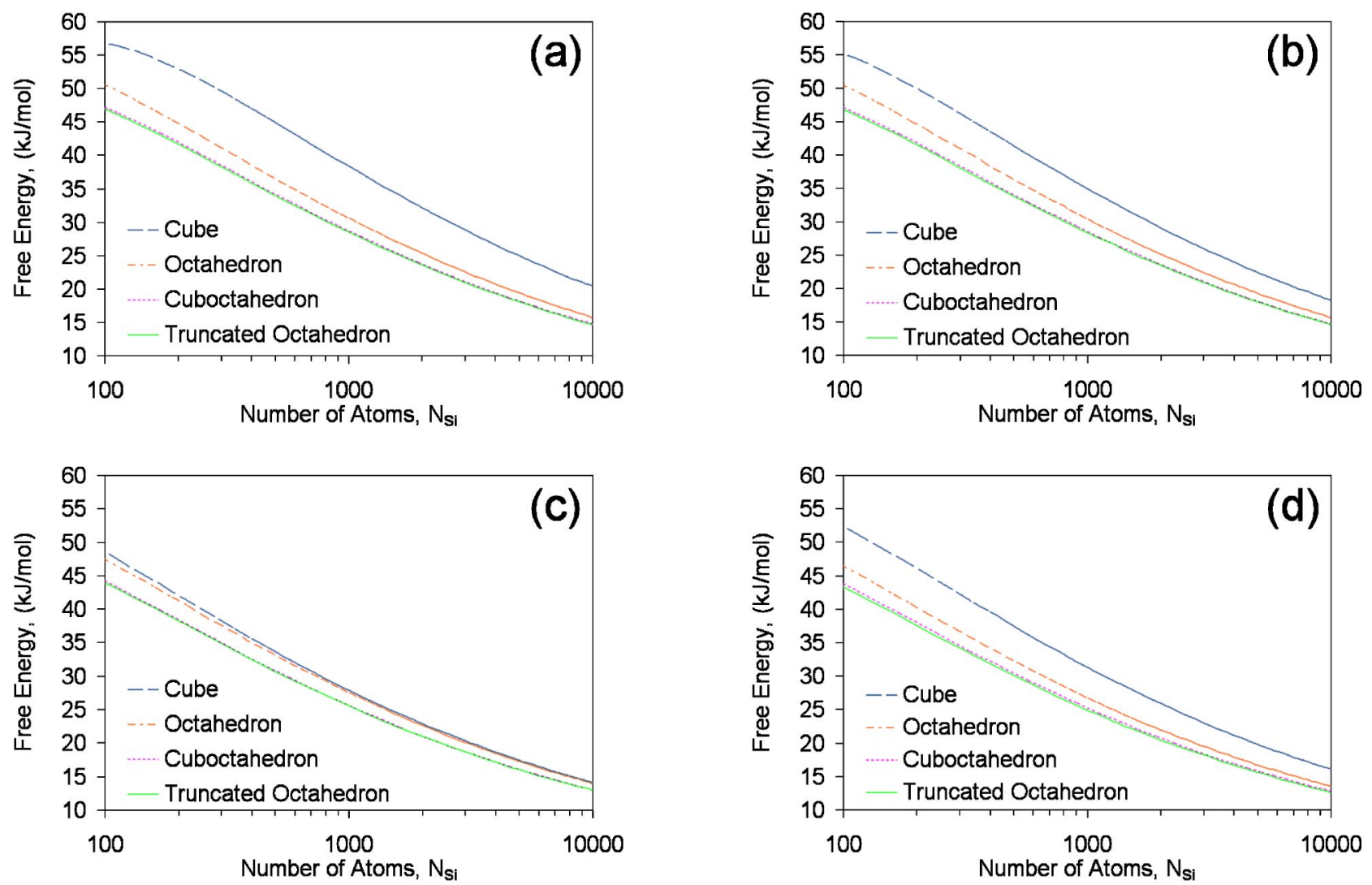

FIG. 2. Comparison of free energies for Si nanoparticles of cube, octahedron, truncated octahedron and cuboctahedron shapes as a function of size using surface energies from (a) LDA (Ref. 24), (b) LDA (Ref. 8) (c) many-body classical potentials (Ref. 25), and (d) experiment (Ref. 17).

work are $7.2 \times 10^{-10} \mathrm{~J} / \mathrm{m}$ for $\mathrm{S}_{A}$ step and $1.9 \times 10^{-9} \mathrm{~J} / \mathrm{m}$ for $\mathrm{S}_{B}$ step. In general however, the Si edge energies for (100) facets and the single steps on diamond and germanium (100) surfaces would produce total edge energies (for crystals containing $10^{3}-10^{6}$ atoms) that are one to three orders of magnitude smaller than the total energies for the adjacent surfaces as listed in Ref. 8.

Since the contribution from the edges and corners is therefore expected to be considerably smaller than that of the surfaces for the sizes larger than $\sim 10^{4}$ atoms, it is assumed that edge and corner energies will have limited significance when considering diamond (silicon, germanium) nanocrystals larger than $\sim 1.2(1.6,1.7) \mathrm{nm}$, and can be neglected over $\sim 5.6(7.3,7.6) \mathrm{nm}$. For crystals smaller than $3 \mathrm{~nm}$ it is more appropriate to perform explicit calculations of isolated structures using density functional theory (DFT) or even tight-binding methods. However, our future efforts include a rigorous investigation of the edge and corner energies of diamond, and the effects these contributions have upon the equilibrium shape.

\section{B. Accuracy of surface energies}

The influence of method used to calculate surface energies on the resulting relative shape stability will now be examined for Si nanocrystals using several available sets of surface energies for comparison. Here we assume $\sigma=\gamma$ and use Laplace-Young model [Eq. (14)], both approximations are to be examined in the following section. In addition to a set of LDA surface energies from Ref. 8, we have employed LDA energies from Ref. 24 as well as a set of experimental data $^{17}$ and a set of energies obtained with classical manybody potentials. ${ }^{25}$ Free energies as a function of nanoparticle size for cube, octahedron, truncated octahedron and cuboctahedron are plotted in Fig. 2. The order of these shapes remains the same for all four sets, truncated octahedron and cuboctahedron being the most stable and nearly degenerate in energy, and the cube being the least stable. The relative energy difference between the shapes is somewhat smaller for the classical potentials set as compared to the other sets. To investigate further the consistency of results for relative energy differences, the difference between energies of the most stable shape, truncated octahedron, and the least stable shape, cube, are plotted in Fig. 3. The results based on LDA (Ref. 8) calculations show the best agreement with results derived from experimental set. Both LDA results have proven applicable for determining relative shape stability if the total free energies of nanoparticles differ by more than $5 \mathrm{~kJ} / \mathrm{mol}$.

\section{Contribution from surface tension}

The choice of technique used to calculate volume dilation $e$ will now be examined in detail, to determine whether Laplace-Young model is applicable in describing elastic energy for non-spherical particles by comparing it to the Stoneham model for cubic and octahedral nanodiamonds. Volume dilations are calculated from expressions (14) and (15) using 


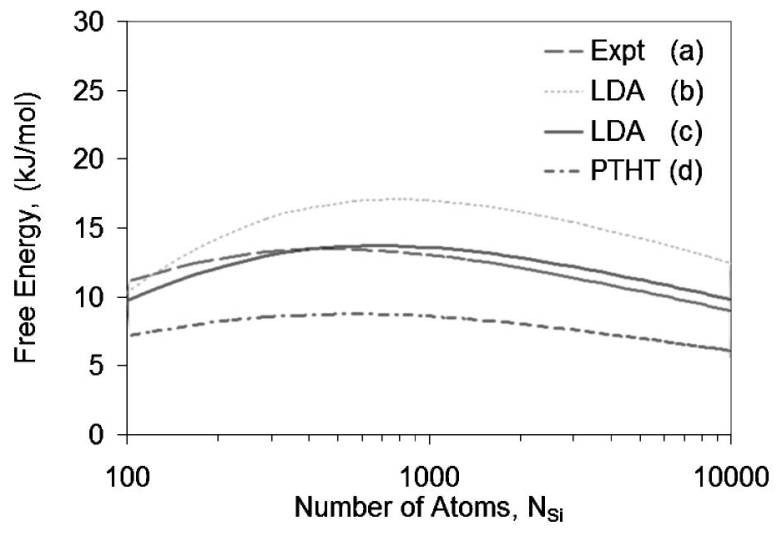

FIG. 3. Comparison of free energy differences between truncated octahedron and cubic shapes of $\mathrm{Si}$ nanoparticles as a function of size calculated using surface energies from (a) experiment (Ref. 17), (b) LDA (Ref. 24), (c) LDA (Ref. 8), and (d) many-body classical potentials (Ref. 25).

experimental values of compressibility $\beta$ (Ref. 26) and the common approximation $\sigma=\gamma$. Using these values in Eq. (12) (ignoring the edge and corner terms) the total free energy was calculated for both models, in addition to results using Eq. (10) for the case where the dilation due to compressive strain is ignored entirely. These results are plotted in Fig. 4 for cubic dehydrogenated (a) and hydrogenated (b) nanodiamonds, and for octahedral dehydrogenated (c) and hydrogenated (d) nanodiamonds. Corresponding energies of diamond nanocrystals explicitly calculated using generalized gradient approximation (GGA) from Ref. 1 are shown in both figures for purposes of comparison. In most cases, both Laplace-Young and Stoneham models are in a close agreement, indicating that for practical purposes the more simple Laplace-Young approach is sufficiently accurate to evaluate the contribution of compressive strain. In the cases when the shape is far from a sphere, with large aspect ratio, for example, one has to consider the Stoneham model for calculation of effective pressure and corresponding volume dilation. The differences between results of explicit DFT GGA calculations ${ }^{1}$ and the present model are small (less than 0.1 $\mathrm{kJ} / \mathrm{mol}$ per atom for the dehydrogenated nanodiamonds, and $0.3 \mathrm{~kJ} / \mathrm{mol}$ per carbon atom for the hydrogenated nanodiamonds) for several different nanocrystal sizes and shapes, therefore validating the model for comparison of stability for different shapes.

The applicability of the $\sigma=\gamma$ approximation was investigated by comparing the results for dehydrogenated $\mathrm{Si}$ with those calculated using more realistic surface tension values. The ratio $\sigma / \gamma$ for $\mathrm{Si}$ (111) was taken from Vanderbilt's LDA calculation ${ }^{27}$ to be 0.87 for [ $\left.2 \times 1\right]$ Pandey-chain reconstruction (average over both directions) and 0.58 for [ $7 \times 7]$ reconstructed analog of dimer adatom stacking fault model. The stress on $\mathrm{Si}(100)[2 \times 1]$ was found to be slighly compressive in one direction and slightly tensile in the other direction. ${ }^{28}$ This anisotropy leads to stress domains on macroscopic (100) facets, ${ }^{28}$ however, the typical size of the domains is about $50 \mathrm{~nm}$, which is larger than the nanoparticle
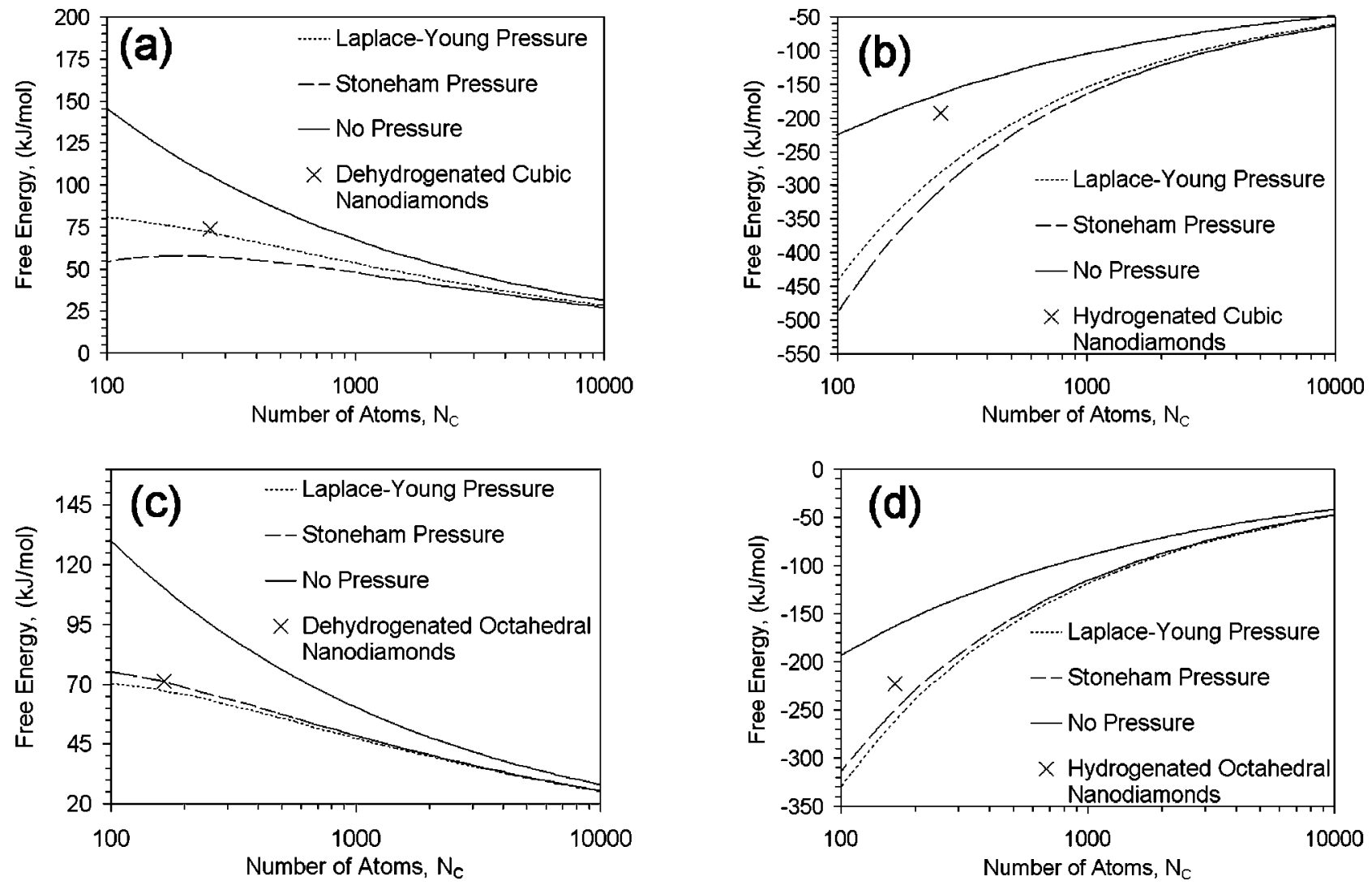

FIG. 4. Influence of compressive strain on total free energy for the (a) dehydrogenated and (b) hydrogenated cubic diamond nanocrystals, and the (c) dehydrogenated and (d) hydrogenated octahedral diamond nanocrystals, as a function of size. The logarithmic $x$-axis spans the sizes approximately 1.2-5.6 $\mathrm{nm}$. The crosses denote the free energy of calculated explicitly using DFT GGA (Ref. 1) for diamond nanocrystals of the corresponding morphology. 


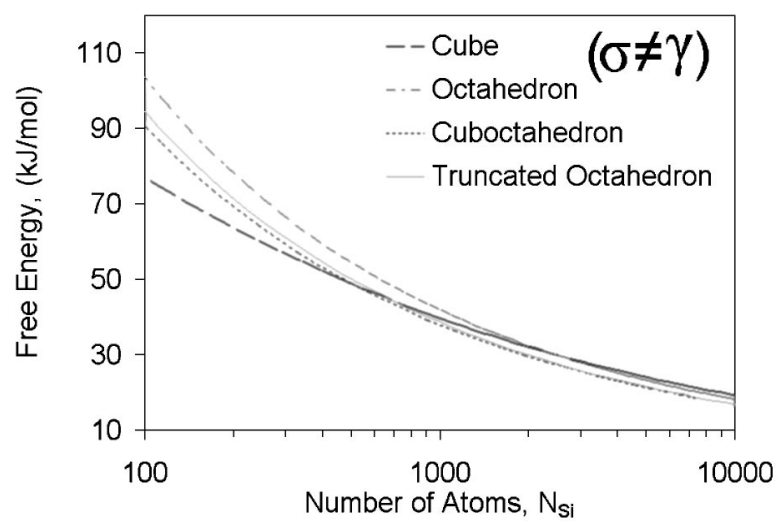

FIG. 5. Comparison of energies of different shapes of dehydrogenated diamond-structured Si nanoparticles as a function of size using LDA values of both surface energies and surface tensions.

size. Thus, the anisotropy was ignored in our consideration The value for $\mathrm{Si}(100)$ surface tension, $\left(\sigma_{x} x+\sigma_{y} y\right) / 2$, was taken from LDA calculations ${ }^{29}$ on $\mathrm{c}[2 \times 1]$ reconstructed surface $(\sigma / \gamma=1.4)$. The results are shown in Fig. 5 for several Si nanocrystal shapes. As compared to Fig. 6(b), there are significant differences in the size range of 100 to 1000 atoms, because the cubic shape is stabilized by a significantly smaller surface tension for (100) surface in this set of data. Several crossovers in stability from shape to shape are observed in this range, the cube going from being the most stable to the least stable as size increases. As expected, no significant changes are exhibited at larger sizes. These results indicate that it is very important to accurately evaluate surface stress for small nanoparticles, since it can be drastically different from the surface energies and have an important contribution to the overall free energy balance.

\section{Predicting morphologies}

The Eq. (12) (ignoring edge and corner effects) with volume dilation calculated from the Laplace-Young model and the surface energies in Ref. 8 for the diamond phase of $\mathrm{C}, \mathrm{Si}$ and $\mathrm{Ge}$ were used to predict the energetically preferred morphology for each species as a function of their size. The surface energy for $\mathrm{Si}(111)$ was changed from [2×1] to $[7 \times 7]$ value once a facet had sufficient size. The results are shown in Fig. 6 for dehydrogenated nanocrystals and in Fig. 7 for hydrogenated nanocrystals as a function of the number of atoms. For dehydrogenated diamond, the most stable morphology at low sizes is predicted to be a sphere, but as size increases cuboctahedron and truncated octahedron become the energetically preferred shapes. Both cubes and octahedrons are significantly higher in energy at sizes up to $\sim 10^{6}$ atoms making them unlikely shapes in this size range.

This situation changes drastically upon hydrogenation. Cubic shape is by far the most stable in hydrogenated nanoparticles of all three species. It is followed by sphere in $\mathrm{Si}$ and $\mathrm{Ge}$, in contrast to diamond, which has octahedron as the next stable shape. This major change in shape behavior upon surface hydrogenation is explained by the large change in surface energies. Thus, the shape of nanoparticles that can be observed experimentally critically depends on adsorbed sur-
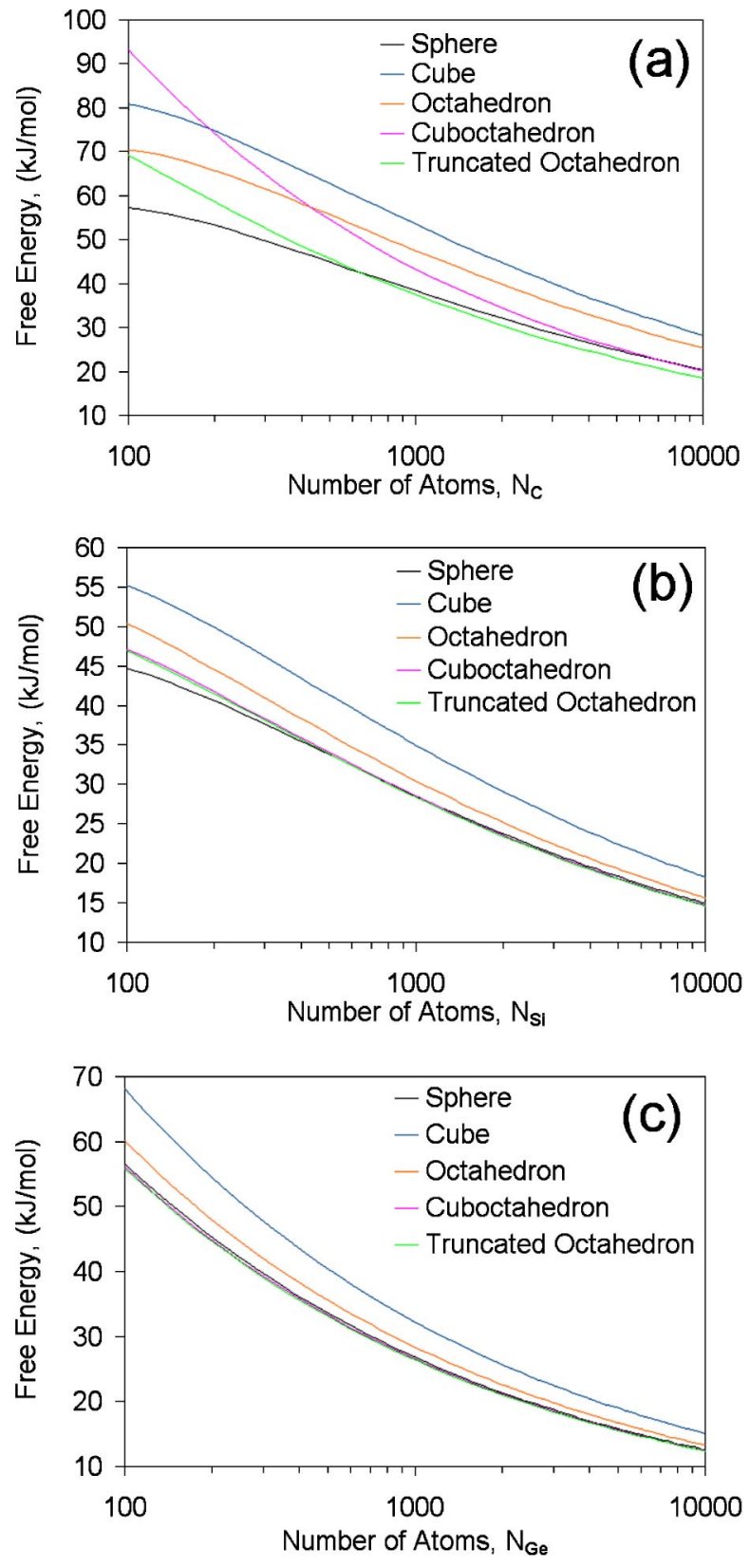

FIG. 6. Comparison of energies of different shapes of dehydrogenated diamond-structured (a) C, (b) Si, and (c) Ge nanoparticles as a function of size. The logarithmic $x$-axis spans the sizes approximately $1.2-5.6 \mathrm{~nm}$ for carbon, $1.6-7.3 \mathrm{~nm}$ for silicon and $1.7-7.6 \mathrm{~nm}$ for germanium nanocrystals.

face species. It is also possible that the observed shape can indicate which species are adsorbed at the surface and perhaps even the degree of surface coverage, since the surface energies are extremely sensitive to such a modification. In general, the energy difference between different shapes decreases with increasing atomic number.

We note here that we have not taken into account highindex surfaces, since there is no consistent set of calculated surface energies for $\mathrm{C}, \mathrm{Si}$, and $\mathrm{Ge}$ available. However, we recognize their importance in determining the shape of nanoparticles, as can be seen, for instance, from numerous observations of (311) facets in $\mathrm{Si}^{13,30} \mathrm{~A}$ complete treatment of nanoparticle shape has to take high-index surfaces into con- 

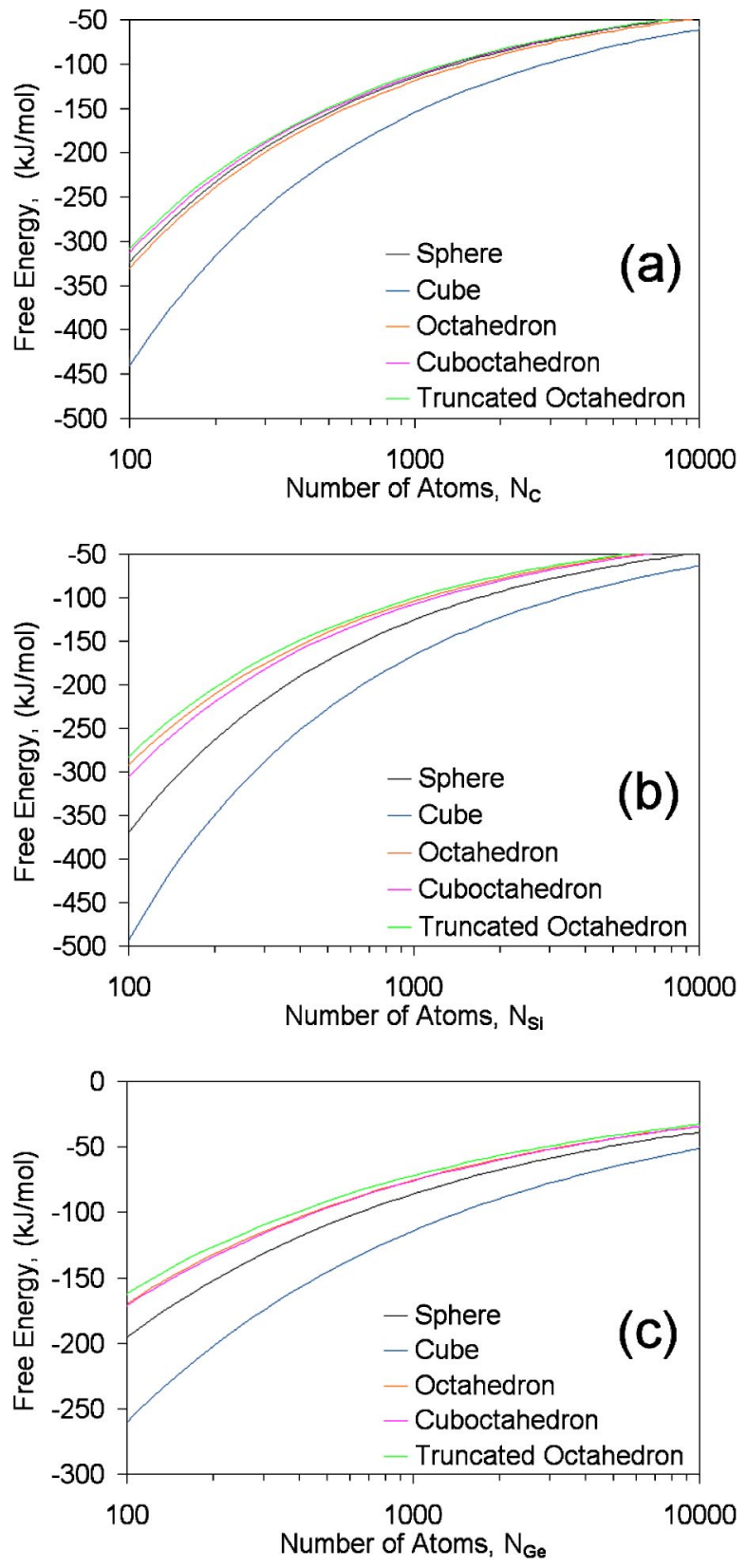

FIG. 7. Comparison of energies of different shapes of hydrogenated diamond-structured (a) C, (b) Si, and (c) Ge nanoparticles as a function of size. The logarithmic $x$-axis spans the sizes approximately $1.2-5.6 \mathrm{~nm}$ for carbon, $1.6-7.3 \mathrm{~nm}$ for silicon, and $1.7-7.6 \mathrm{~nm}$ for germanium nanocrystals.

(a)

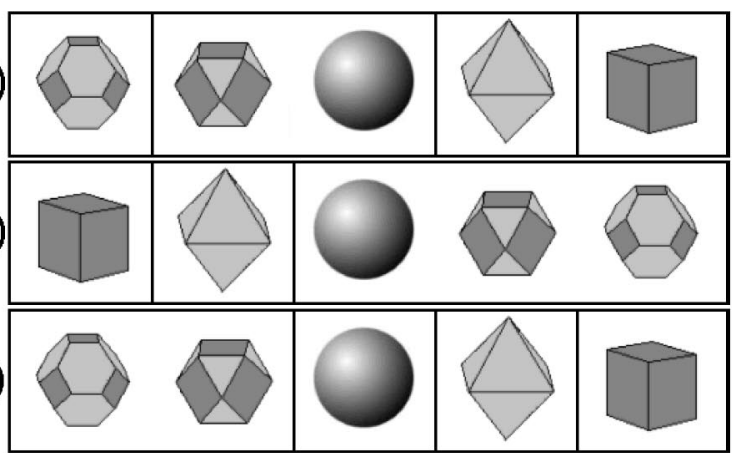

sideration, which can be done in a straightforward manner using computational methods.

The order of preferred morphologies is shown in Fig. 8 for the size of $\geqslant 10000$ atoms for all three semiconductors, for both hydrogenated and dehydrogenated nanocrystals. It is important to note that in the case of dehydrogenated nanodiamond, the thermodynamically most stable phase of carbon for particles smaller than $\sim 1.9 \mathrm{~nm}$ is fullerenes, ${ }^{31}$ ad bucky diamonds may be expected up to $\sim 2.2 \mathrm{~nm},{ }^{32}$ both of which our model is not designed to describe. For dehydrogenated $\mathrm{Si}$ and $\mathrm{Ge}$, octahedron, truncated octahedron and sphere are very close energetically in all size ranges, making appearance of these shapes quite likely. For example, the "rounded" tetrakaidecahedron shape of the nanoscale voids in Si observed by Eaglesham et al. ${ }^{17}$ is virtually the same as the truncated octahedron that we predict to be the preferred shape for Si nanocrystals in this size range. It should be noted that comparison of our results to the experimental data is meaningful only if the experimental shapes are the thermodynamically most stable shapes. It is often the case that kinetic processes of nanoparticle synthesis result in nonequilibrium structures, which should be described using kinetic models rather than purely thermodynamic stability arguments.

\section{CONCLUSION}

Summarizing, a thermodynamic model was developed to describe the transformation of nanocrystal shape as a function of its size. It takes into consideration surface energies and stresses, as well as edge and corner energies, which can be calculated by nonempirical methods. This model can be used for any type of material, any crystallographic system and can be used to predict phase transitions as a function of shape and size by equating free energies for the most stable shapes of opposing phases. The model has been applied to the diamond phase of group IV semiconductors, namely, C, $\mathrm{Si}$, and Ge. We have shown that Laplace-Young model is satisfactory to describe volume dilation caused by surface tension, and predict that edge and corner contributions to the total energy rapidly diminish with increase in nanocrystal size, becoming unimportant at sizes above $\sim 10000$ atoms. It was shown that accurate evaluation of surface stress is important at sizes below $\sim 1000$ atoms to properly account for elastic energies, and was demonstrated that predictions based

FIG. 8. Predicted sequence of the preferred shapes (left to right) for dehydrogenated diamond (a), silicon (b) and germanium (c), and hydrogenated diamond (d), silicon (e) and germanium (f) nanoparticles of $\geqslant 10000$ atoms. Energetically undistinguishable shapes are not separated by a line. 
on density functional results are in agreement with predictions based on experimental surface energies.

Thermodynamically favored morphology of diamond nanoparticles was predicted. These predicted results have been compared with those obtained from explicit DFT GGA calculations of various nanoparticles, ${ }^{1}$ and contrasted with the predicted morphologies of $\mathrm{Si}$ and $\mathrm{Ge}$ nanoparticles. Finally, by comparing energetics of hydrogenated and dehydrogenated nanocrystals it was shown that adsorbed species are a dominant factor in determining their shape. Thus, this model can be useful in comparing stability of different nanoparticles as a function of their phase, size, shape, and surface coverage by various adsorbates.

\section{ACKNOWLEDGMENTS}

This work has been supported by the U.S. Department of Energy, BES-Chemical Sciences, under Contract No. W-31109-ENG-38. The authors would also like to acknowledge Larry A. Curtiss for useful discussions.

${ }^{1}$ A. S. Barnard, S. P. Russo, and I. K. Snook, Diamond Relat. Mater. 12, 1867 (2003).

${ }^{2}$ A. S. Barnard, S. P. Russo, and I. K. Snook, Nano Lett. 3, 1323 (2003).

${ }^{3}$ F. Lacona, G. Franzò, and C. Spinella, J. Appl. Phys. 87, 1295 (2000).

${ }^{4}$ G. F. Grom, D. J. Lockwood, J. P. McCaffrey et al., Nature (London) 407, 358 (2000).

${ }^{5}$ W. E. Buhro and V. I. Cowin, Nat. Mater. 2, 138 (2003).

${ }^{6}$ A. Puzder, A. J. Williamson, F. A. Reboredo, and G. Galli, Phys. Rev. Lett. 91, 157405 (2003).

${ }^{7}$ L. Pizzagalli, G. Galli, J. E. Klepeis, and F. Gygi, Phys. Rev. B 63, 165324 (2001).

${ }^{8}$ A. A. Stekolnikov, J. Furthmüller, and F. Bechstedt, Phys. Rev. B 65, 115318 (2002)
${ }^{9}$ A. M. Stoneham, J. Phys. C 10, 1175 (1977).

${ }^{10}$ G. Wulff, Z. Kristallogr. 34, 449 (1901).

${ }^{11}$ C. Herring, Phys. Rev. 82, 87 (1951).

${ }^{12}$ H. P. Bonzel, Prog. Surf. Sci. 67, 45 (2001).

${ }^{13}$ H.-C. Jeong and E. D. Williams, Surf. Sci. Rep. 34, 171 (1999).

${ }^{14}$ C. L. Cleveland and U. Landman, J. Chem. Phys. 94, 7376 (1991).

${ }^{15}$ M. José-Yacamán, J. A. Ascencio, H. B. Liu, and J. Gardea-Torresdey, J. Vac. Sci. Technol. B 19, 1091 (2001).

${ }^{16}$ N. Moll, M. Scheffler, and E. Pehlke, Phys. Rev. B 58, 4566 (1998).

${ }^{17}$ D. J. Eaglesham, A. E. White, L. C. Feldman, N. Moriya, and D. C. Jacobson, Phys. Rev. Lett. 70, 1643 (1993); 72, 1392 (1994); 72, 2975 (1994).

${ }^{18}$ A. P. Alivisatos, J. Phys. Chem. 100, 13226 (1996).

${ }^{19} \mathrm{~T}$. Tyler (private communication). Figure 1 shows a Molybdenum wire that was etched in $\mathrm{KOH}$ at $+12 \mathrm{VDC}$, with detonation nanodiamond deposited by pulsed electrophoresis from diamond-ethanol suspension ( $2 \mathrm{~g}$ of diamond per 11 of ethanol, the suspension was sonicated for $15 \mathrm{~min}$ prior to deposition). The radius of curvature at tip apex is $\sim 60 \mathrm{~nm}$.

${ }^{20}$ G. Kern and J. Hafner, Phys. Rev. B 58, 2161 (1998).

${ }^{21}$ M.-H. Tsai and Y.-Y. Yeh, Phys. Rev. B 58, 2157 (1998).

${ }^{22}$ H. J. W. Zandvliet, Phys. Rev. B 61, 9972 (2000).

${ }^{23}$ H. J. W. Zandvliet, Phys. Rep. 388, 1 (2003).

${ }^{24}$ S. Hong, J. Korean Phys. Soc. 37, 93 (2000).

${ }^{25}$ H. Balamane, T. Halicioglu, and W. A. Tiller, Phys. Rev. B 46, 2250 (1992).

${ }^{26}$ Calculated using the expression $\beta=1 / B_{0}$, where $B_{0}^{C}=443 \mathrm{GPa}, B_{0}^{S i}$ $=98 \mathrm{GPa}$, and $B_{0}^{G e}=75 \mathrm{GPa}$ were obtained from the Handbook of Chemistry and Physics, edited D. R. Lide, 84th ed. (CRC Press, Boca Raton, 2003).

${ }^{27}$ D. Vanderbilt, Phys. Rev. Lett. 59, 1456 (1987).

${ }^{28}$ O. L. Allerhand, D. Vanderbilt, R. D. Meade, and J. D. Joannopoulos, Phys. Rev. Lett. 61, 1973 (1988).

${ }^{29}$ A. Garcia and J. E. Northrup, Phys. Rev. B 48, 17350 (1993).

${ }^{30}$ Y.-N. Yang and E. D. Williams, J. Vac. Sci. Technol. A 8, 2481 (1990).

${ }^{31}$ A. S. Barnard, S. P. Russo, and I. K. Snook, J. Chem. Phys. 118, 5094 (2003).

${ }^{32}$ A. S. Barnard, S. P. Russo, and I. K. Snook, Phys. Rev. B 68, 073406 (2003). 